\title{
The autonomic nervous system in patients with spinal cord lesion
}

\author{
JJ Wyndaele, Editor \\ Antwerp University Hospital, Antwerp, Belgium \\ E-mail: spinalcord@uza.be
}

Dear Spinal Cord reader,

This issue, October 2008, contains several manuscripts related to the autonomic nervous system. Autonomic dysfunction is a major and not as yet fully understood consequence of spinalcord injury. It can be the cause of very serious disabilities and requires much more research. It will, without doubt, become a special focus of treatment strategies in the future. Bladder, cardiovascular, bowel and sexual dysfunctions after spinal-cord injury, all relate to the changes within the autonomic nervous system control of these functions. The negative impact of, for instance, profuse sweating, sudden changes in blood pressure, and urinary and faecal incontinence is huge. For patients, the dysfunction can be experienced as a pounding headache, a tingling sensation on the face and neck, blotchy skin around the neck and goose bumps. They are terrible, frightening and sometimes life threatening. The search for treatment options for the different conditions fortunately continues. The attention to autonomic dysfunctions is growing; the need for standardization is often felt. We expect to publish major reports on this in the coming months.

This month's review article, by Gillis et al., deals with the non-pharmacological management of orthostatic hypotension after spinal-cord injury. It evaluates whether compression to the abdominal region, upper body exercise, functional electrical stimulation applied to the legs and biofeedback are useful. The capacity of the sympathetic nervous system to respond to a cardiovascular challenge in human spinal-cord injury is studied in an original article by Brown and Macefield with inspiratory-capacity apnoea. Whether selective activation of muscle and skin nociceptors triggers exaggerated sympathetic responses in spinal-cord injury patients is a third important study, by Burton et al., and is also included in this issue.

A urologic study on neuropathic bladder dysfunction in patients with motor complete and sensory incomplete spinal cord lesion, by Rapidi et al., and a study on the sexual life of males over the age of 50 years with spinal cord lesions of at least 20 years by Lombardi et al. provide interesting observations.

The way of life for adults with tetraplegia due to trauma creates worries in patients and relatives, in carers and health authorities. The study of Bergmark et al. provides determinants for residence and quality of life.

Spinal cord lesion-related coping strategies and emotional well-being can be explored by questionnaires. Migliorini et al. translated SCL CSQ and SCL EWQ and made an Australian validation.

An original article by Hua Guan et al. provides an estimate of the motor unit number of the tibialis anterior muscle in normal subjects and patients with spinal-cord injury. Two interesting case reports on intramedullary melanotic schwannoma (Mouchaty et al.) and on isolated intraspinal germinoma in a child with congenital adrenal hyperplasia (Tubbs et al.) conclude this October issue.

Spinal Cord (2008) 46, 651; doi:10.1038/sc.2008.119 